\title{
PÉREZ Patrick, Les Indiens hopi d'Arizona. Six études anthropologiques
}

\section{Emmanuel Désveaux}

\section{OpenEdition \\ Journals}

Édition électronique

URL : https://journals.openedition.org/jsa/1800

DOI : 10.4000/jsa. 1800

ISSN : 1957-7842

\section{Éditeur}

Société des américanistes

\section{Édition imprimée}

Date de publication : 5 juin 2004

Pagination : 205-207

ISSN : 0037-9174

\section{Référence électronique}

Emmanuel Désveaux, «pérez Patrick, Les Indiens hopi d'Arizona. Six études anthropologiques 》, Journal de la Société des américanistes [En ligne], 90-2 | 2004, mis en ligne le 17 novembre 2005, consulté le 04 septembre 2022. URL : http://journals.openedition.org/jsa/1800 ; DOl : https://doi.org/10.4000/jsa. 1800

Ce document a été généré automatiquement le 4 septembre 2022

Tous droits réservés 


\title{
PÉREZ Patrick, Les Indiens hopi d'Arizona. Six études anthropologiques
}

\author{
Emmanuel Désveaux
}

\section{RÉFÉRENCE}

PÉREZ Patrick, Les Indiens hopi d'Arizona. Six études anthropologiques, L'Harmattan,

coll. « Inter-national », Paris, 2004, 162 p., bibl., chronologie

1 Le recueil d'articles est un genre que les éditeurs fuient aujourd'hui comme la peste. Pourtant Patrick Pérez, architecte et ethnologue, enseignant à l'école d'architecture de Toulouse, a réussi à convaincre l'un d'entre eux de se lancer dans l'aventure. Il y a tout lieu de s'en réjouir car le résultat est un petit ouvrage de 150 pages qui fait parfaitement office d'introduction aux études hopi. Cet effet de didactisme tient au fait que l'origine des textes rassemblés ici se trouve pour l'essentiel dans des conférences qui s'adressaient à un public de non-spécialistes. Mais, plus profondément, on pressent chez Pérez un souci particulier d'écriture. Aussi la précision et l'élégance de cette dernière contribuent-elles largement à cette impression d'intelligibilité élargie qui se dégage de l'ensemble.

2 La plupart des grands thèmes de l'ethnologie des Pueblos est abordée : l'archéologie, à propos des Anasazi, et l'histoire, à travers la question du traitement passé et présent des nombreux vestiges architecturaux de cette région; la technique et le rituel, ces deux thèmes indissociables renvoyant toujours, comme Pérez le précise à juste titre, à celui de la différence sexuelle. L'auteur prête une attention particulière au paysage - c'était d'ailleurs le thème principal de sa thèse soutenue en 1998 -, au détriment de la dimension purement architecturale de la civilisation pueblo. Ce qui peut surprendre de la part d'un architecte, mais se comprend mieux lorsqu'on sait l'immense corpus déjà consacré au sujet... L'auteur s'est en effet particulièrement attaché à montrer la lecture calendaire du paysage, en référence à la course du soleil. Les Hopi entretenaient une classe de prêtres chargés d'observer quotidiennement le point d'élévation du soleil sur la ligne d'horizon et d'en déduire la date de telles ou telles manifestations rituelles. Pérez souligne avec 
justesse que seule la sédentarité la plus rigoureuse autorise une telle pratique. Dans ce cas, les repères topographiques restent constants. À l'opposé, les peuples plus ou moins nomades, faute de tels repères, doivent se «contenter " des lunaisons afin de mesurer l'écoulement temporel. Encore faut-il que le paysage soit dépourvu de couvert végétal, ce qui est typiquement le cas des semi-désert d'Arizona, et que les postes d'observation soient en hauteur, ce qui est bien sûr le cas des mesa sur lesquelles sont bâtis depuis fort longtemps les villages. Incidemment, Pérez nous convainc facilement que les Pueblos se rattachent à la Mésoamérique, sous ce double rapport du calendrier et de la sédentarité.

De nombreuses remarques de même nature justifient pleinement la fréquentation de ce petit volume. Nous n'en retiendrons qu'une. Elle nous a particulièrement frappé. On sait que les Pueblos ont agi très vite contre la présence d'ethnographes. Dès la fin du $\mathrm{XIX}^{\mathrm{e}}$ siècle, ils leur restreignent en effet très sévèrement l'accès à tout ce qui touche à la sphère du rituel, déclaré comme relevant d'un ordre du secret. En réalité, à ce momentlà, le mal était fait, Fewkes et d'autres ayant déjà diffusé leurs matériaux ethnographiques dans diverses publications dont les fameux rapports du Bureau of Ethnology de la Smithsonian. Le drame des Pueblos n'était pas tant que des secrets rituels soient divulgués aux Blancs, mais que soit abolie par leur faute la compartimentation de la connaissance cérémonielle, répartie entre les différentes confréries, et qui était au fondement de leur organisation rituelle, laquelle valait également d'organisation sociale.

La présentation de l'univers hopi que nous offre Pérez combine, pour notre plus grand bonheur, l'hyperculturalisme d'un Worf (pour lequel les Hopi étaient, rappelons-le, la principale référence ethnographique lorsqu'il ébauchait, en compagnie de Sapir, sa fameuse hypothèse d'une relation étroite entre langue et culture) et un dualisme de bon aloi directement inspiré du structuralisme lévi-straussien. Cela étant, il faut se rendre à l'évidence que cette association fonctionne sans soulever de difficultés majeures uniquement parce qu'elle exclut toute perspective comparatiste. Et c'est un peu le reproche que l'on pourrait formuler à Pérez. Ses Pueblos, ses Hopi surtout, apparaissent comme des isolats. Le reste de l'horizon anthropologique amérindien est virtuellement inexistant pour lui. En ce sens, les propos de Pérez se coulent trop bien dans le moule des études sur les Pueblos, alors même qu'il est urgent de faire rupture avec elles sur ce point. Les Pueblos ne sont pas des OVNI atterris par hasard au cœur des déserts du SudOuest nord-américain!

\section{AUTEURS}

\section{EMMANUEL DÉSVEAUX}

EHESS/Musée du quai Branly, Paris 